\title{
Elimination of Dendritic Spines with Long-Term Memory Is Specific to Active Circuits
}

\author{
Jeff Sanders, ${ }^{1}$ Kiriana Cowansage, ${ }^{2}$ Karsten Baumgärtel, ${ }^{2}$ and Mark Mayford ${ }^{2}$ \\ ${ }^{1}$ The Stein Institute for Research on Aging, University of California San Diego, La Jolla, California 92093-0603, and ${ }^{2}$ Dorris Center for Neuroscience, The \\ Scripps Research Institute, Department of Cell Biology, La Jolla, California 92037-1000
}

Structural changes in brain circuits active during learning are thought to be important for long-term memory storage. If these changes support long-term information storage, they might be expected to be present at distant time points after learning, as well as to be specific to the circuit activated with learning, and sensitive to the contingencies of the behavioral paradigm. Here, we show such changes in the hippocampus as a result of contextual fear conditioning. There were significantly fewer spines specifically on active neurons of fearconditioned mice. This spine loss did not occur in homecage mice or in mice exposed to the training context alone. Mice exposed to unpaired shocks showed a generalized reduction in spines. These learning-related changes in spine density could reflect a direct mechanism of encoding or alternately could reflect a compensatory adaptation to previously described enhancement in transmission due to glutamate receptor insertion.

\section{Introduction}

Dendritic spines are the primary sites for excitatory synaptic contact on pyramidal neurons in the brain. The morphology and density of dendritic spines are both altered in neuropsychiatric conditions that affect cognitive function (Penzes et al., 2011). The spine abnormalities found in these illnesses may underlie cognitive impairment by interfering with the intricate structural plasticity that has been observed for dendritic spines. For instance, the size and density of spines have been found to change in a number of synaptic and behavioral plasticity paradigms, leading to the suggestion that they may form a structural basis for longterm memory (Moser et al., 1994; Trachtenberg et al., 2002; Leuner et al., 2003; Holtmaat et al., 2005; Yang et al., 2008; Kelsch et al., 2009; Restivo et al., 2009; Lai et al., 2012).

Spine changes that result from long-term potentiation (LTP) and long-term depression (LTD) suggest an important role for neural activity in synaptic modifications that may be important for long-term memory. LTP leads to a proliferation of spines, while LTD is conversely associated with spine elimination (Desmond and Levy, 1986; Trommald et al., 1996; Bastrikova et al., 2008). Whether spine changes exist on the active circuits that are proposed to subserve long-term memory, however, is unclear (De Roo et al., 2008). Spine proliferation as a result of LTP tends to be transient, reverting to baseline values shortly after LTP induction (De Roo et al., 2008; Wosiski-Kuhn and Stranahan,

\footnotetext{
Received March 2, 2012; revised July 1, 2012; accepted July 19, 2012.

Author contributions: J.S. and M.M. designed research; J.S., K.C., K.B., and M.M. performed research; J.S. and M.M. analyzed data; J.S. wrote the paper.

This work was supported by National Institutes of Health Grants DA028300, MH057368, and MH019334.

The authors declare no competing financial interests.

Correspondence should be addressed to Mark Mayford, Dorris Neuroscience Center, The Scripps Research

Institute, Department of Cell Biology, 10550 N. Torrey Pines Road, La Jolla, CA 92037-1000. E-mail: mmayford@scripps.edu.

DOI:10.1523/JNEUROSCI.1131-12.2012

Copyright $\odot 2012$ the authors $\quad 0270-6474 / 12 / 3212570-09 \$ 15.00 / 0$
}

2012). This observation challenges the proposition that there are durable and activity-specific changes in spine number that are characteristic of long-term memory.

To study whether those neurons active at learning exhibit an enduring spine change with long-term memory, we used a transgenic mouse, GFP-GluR $1^{\mathrm{c}-f o s}$. This mouse expresses a long-lasting dendritic marker (GFP-GluR1) of neural activity, allowing us to assess structural changes in neurons activated at the time of learning relative to inactive neurons in the CA1 region of the hippocampus. We found that spines on active neurons of fear-conditioned animals, but not homecage animals or animals exposed to context alone or unpaired shock, were significantly reduced $24 \mathrm{~h}$ following training relative to spines on inactive neurons. These data point to a mechanism of enduring activity-related synaptic refinement as a component of long-term memory.

\section{Materials and Methods}

Animals. GFP-GluR1 ${ }^{\text {c-fos }}$ mice were generated and genotyped as previously described (Matsuo et al., 2008). All colony maintenance and procedures were conducted according to The Scripps Research Institute guidelines for the humane care and use of laboratory animals. Animals were bred on a $12 \mathrm{~h}$ light/dark cycle and were provided with food and water ad libitum. Animals carrying both the c-fos-tetracycline transactivator (tTA) and GFP-GluR1 transgenes were used for experiments. Mice were kept off of doxycycline (dox) during postnatal development and placed on dox $(40 \mathrm{mg} / \mathrm{kg})$ at weaning. Prior data from our laboratory have shown that GFP-GluR1 is degraded within 2 weeks of GFP-GluR $1^{\text {c-fos }}$ mice being placed on dox, allowing us to eliminate any expression from the early postnatal period with this protocol (Matsuo et al., 2008). Only males were used since estrogen is known to affect dendritic spine density (Murakami et al., 2006). Experimental mice were 2-4 months of age.

Learning protocol. Four experimental groups of GFP-GluR1 $1^{\mathrm{c}-\text { fos }}$ mice were used: homecage control (HC) $(n=9$ mice); contextual control training (CT) $(n=10$ mice); contextual fear conditioning (FC) $(n=11$ 
A
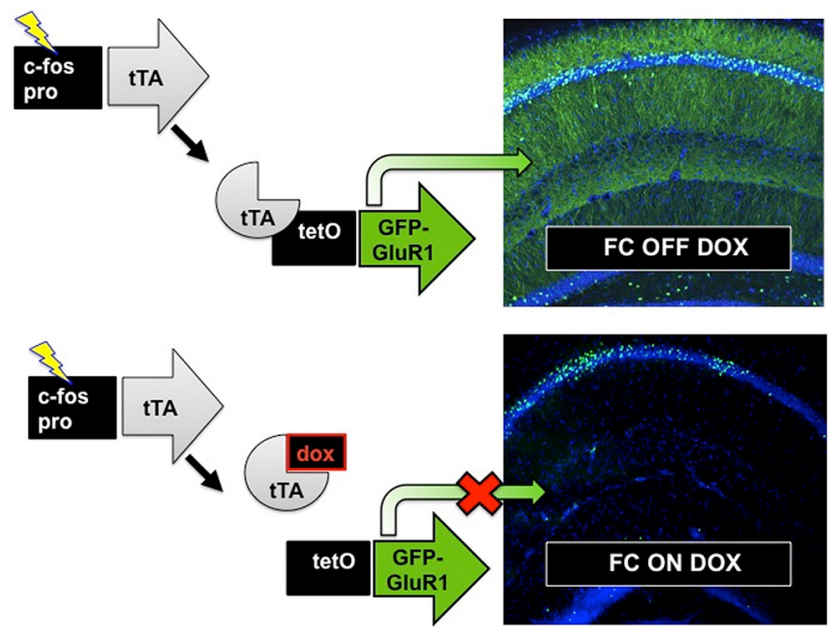

B

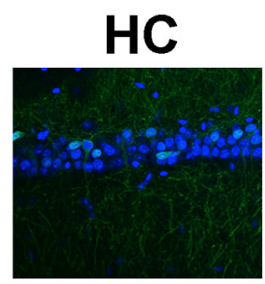

FC

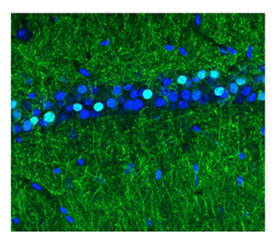

C

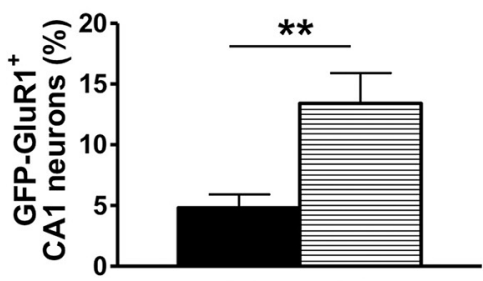

HC

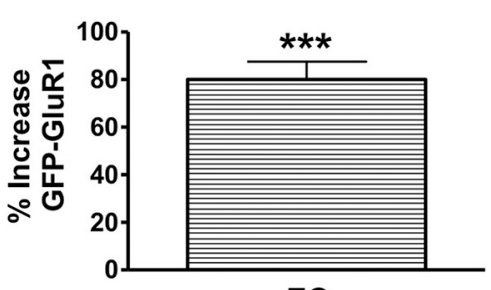

FC

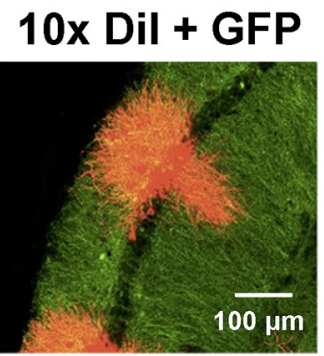

$60 \times$ Dil

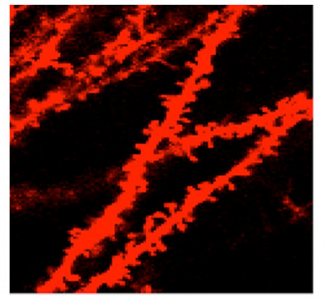

60x Dil + GFP

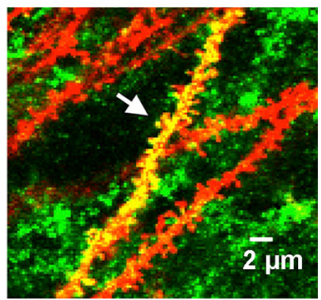

D

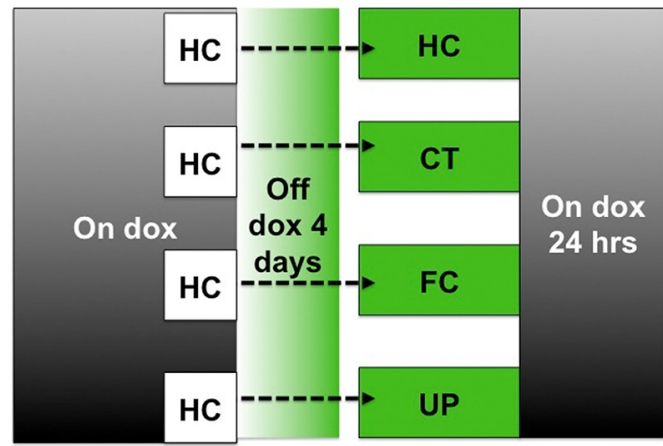

Perfuse

and

analyze

Figure 1. Method for identifying active circuits with GFP-GluR1 $1^{\text {c-fos }}$ mice. $A$, Diagram of the transgenic system. The c-fos promoter is used to drive tTA that in turn activates expression of GFP-GluR1 from the tet0 promoter. Dietary doxycycline prevents activation of GFP-GluR1, allowing the time of active circuit labeling to be experimentally controlled. $\boldsymbol{B}$, Immunohistochemistry confirmed expression of GFP-GluR1 with fear conditioning that is above home cage levels. FC resulted in a threefold increase in the percentage of CA1 neurons with cytoplasmic GFP. ${ }^{* *} p<0.01$. Data are the mean percentage of total CA1 neurons \pm SEM. There was an $\sim 80 \%$ increase in GFP signal within dendritic layers of the hippocampal CA1 region. ${ }^{* * *} p<0.001$, significantly different

mice); and unpaired shock training (UP) $(n=$ 8 mice). Mice were individually housed for $2 \mathrm{~d}$ and handled for 2 min, twice per day, during this time. Mice were then taken off of dox for $4 \mathrm{~d}$ before training. Studies in our laboratory have found that this period is sufficient to allow full clearance of dox.

FC involved placing mice in a wintergreenscented square chamber with black-and-white checkered pattern and aluminum walls $(30 \mathrm{~cm}$ length $\times 24 \mathrm{~cm}$ width) and a grid floor that delivers footshocks (FreezeFrame). An FC session consisted of $120 \mathrm{~s}$ of free exploration followed by four nonsignaled footshocks ( $\mathrm{du}$ ration $2 \mathrm{~s}$, intensity $0.7 \mathrm{~mA}$ ) with an interstimulus interval of $60 \mathrm{~s}$. Mice remained in the chamber for $60 \mathrm{~s}$ after the last shock. We performed two FC sessions that were separated by $2 \mathrm{~h}$. CT mice were subject to the same procedure without shock.

UP training was conducted as outlined previously (Matsuo et al., 2008). We first placed mice in a novel chamber (context A: square plastic chamber surrounded with white walls, plastic floor with sani-chips and wintergreen scent) for $360 \mathrm{~s}$ without footshocks. After context A exposure, mice were placed in their home cage, and $20 \mathrm{~min}$ later they underwent four nonsignaled footshocks (duration $2 \mathrm{~s}$, intensity $0.7 \mathrm{~mA}$ ) with an interstimulus interval of $4 \mathrm{~s}$. These shocks began immediately after mice were placed in the conditioning chamber (context B: rectangular chamber, Plexiglas front and back with black-and-white checkered pattern and aluminum side walls, with a grid floor), and mice were immediately returned to their home cages after the last shock. We performed two UP sessions that were separated by $2 \mathrm{~h}$. HC animals remained in their cage during these experimental procedures.

Immediately after the final experimental treatment, mice from the $\mathrm{HC}, \mathrm{CT}, \mathrm{FC}$, and UP groups were placed on a high-dose dox diet (1 $\mathrm{g} / \mathrm{kg}$ ) overnight to suppress further activation of the GFP-GluR1 transgene. Studies in our laboratory have confirmed that overnight feeding with high-dose dox will block transactivation.

Tissue preparation. Twenty-four hours after fear conditioning, mice used for dendritic spine analysis were anesthetized with isofluorane and rapidly perfused with cold $1 \times \mathrm{PBS}$ for $1.5 \mathrm{~min}$. This was followed by $3.5 \mathrm{~min}$ of perfusion with cold $4 \%$ paraformaldehyde (PFA). Brains were then removed and incubated for $1 \mathrm{~h}$ in cold $4 \%$ PFA and transferred to $30 \%$ sucrose where they were stored at $4^{\circ} \mathrm{C}$ overnight. The next day, $100 \mu \mathrm{m}$ sections were obtained on a vibratome and sections collected in

$\leftarrow$

from HC. Data are the mean percentage increase GFP intensity \pm SEM (HC, $n=6$ mice; FC, $n=6$ mice). C, Confocal image of hippocampus labeled with GFP (green) and Dil (red). A $60 \times$ confocal image reveals dendrites of active neurons that colocalized GFP with Dil (yellow) (designated by arrow) versus dendrites from inactive cells (red). D, Diagram of experimental design. 
cold $1 \times$ PBS. A separate experiment that assessed the induction of GFP-GluR1 to FC used fresh $100 \mu \mathrm{m}$ sections that were fixed in cold 4\% PFA in PBS for $1 \mathrm{~h}$.

Immunohistochemistry. Immunohistochemistry was performed as described previously (Matsuo et al., 2008). Sections were incubated in $5 \%$ BSA in PBS at room temperature (RT) for $30 \mathrm{~min}$. Rabbit-anti-GFP (1:2000) was then added and sections were allowed to incubate at $4^{\circ} \mathrm{C}$ overnight, washed $3 \times 5 \mathrm{~min}$ in PBS at RT, and incubated in Alexa Fluor 488 goat-antirabbit secondary antibody $(1: 800)$ at $4^{\circ} \mathrm{C}$ overnight. After this incubation, they were washed $3 \times 5 \mathrm{~min}$ in PBS at RT and stained with 1,1'-dioctadecyl-3,3,3'3' -tetramethylindocarbocyanine perchlorate (DiI). Experiments that assessed GFP-GluR1 induction to FC used immunohistochemistry as described above with the addition of $0.15 \%$ Triton $\mathrm{X}-100$ for all incubations. This allowed us to visualize the cytoplasmic expression of GFP-GluR1 to quantify the number of CA1 neurons activated by FC.

DiI. DiI labeling was performed as described previously (Matsuo et al., 2008). Micropipettes were coated with DiI dissolved in ethanol at 10 $\mathrm{mg} / \mathrm{ml}$. The tips of DiI-coated micropipettes were then inserted at several positions in the hippocampal CA1 pyramidal cell layer of fixed slices. Slices were placed in RT PBS for $2 \mathrm{~h}$ to allow the DiI to spread throughout the dendritic arbor. Sections were mounted with SlowFade mounting medium (Invitrogen) and stored at $4^{\circ} \mathrm{C}$.

Data acquisition and analysis. To verify the induction of GFP-GluR1 to FC, we used confocal microscopy to measure the number of CA1 neurons with cytoplasmic expression of GFP-GluR1. The number of cells with cytoplasmic GFPGluR1 expression was quantified relative to the total population stained with TOPRO-3. The intensity of GFP staining was measured in the hippocampal CA1 dendritic layers of $\mathrm{HC}$ versus FC mice.

Spines were imaged with confocal microscopy within 1 week of mounting sections. Dendrites were randomly imaged using an oilimmersion $60 \times$ objective with $2 \times$ zoom, and the experimenter was blind to the experimental conditions. Images were acquired using sequential imaging with an argon $(488 \mathrm{~nm})$ and $\mathrm{HeNe}(543 \mathrm{~nm})$ laser. To minimize quenching of fluorescence $z$-stacks of 5-8 $\mu \mathrm{m}$ thickness consisting of sections at $0.12 \mu \mathrm{m}$ increments were rapidly scanned within a $107 \times 107 \mu \mathrm{m}$ imaging area. We coupled our sampling of segments from active and inactive neurons within each sampled region. This was to minimize any regional differences in dendrite structure as a source of variability in comparing active versus inactive circuits. A represented image of active segments that were coupled with the sampling of inactive segments within the same image is depicted (Fig. 1C). In order for a dendrite to be sampled, it had to have consistently bright and continuous DiI labeling throughout its course. It also had to be easily separated from neighboring dendrites to avoid obstruction of spine counts. Primary dendrites were not included in the analysis.

Images were analyzed with Neurolucida software (MBF Biosciences) while blinded to experimental conditions. Dendrites that were strongly dou-

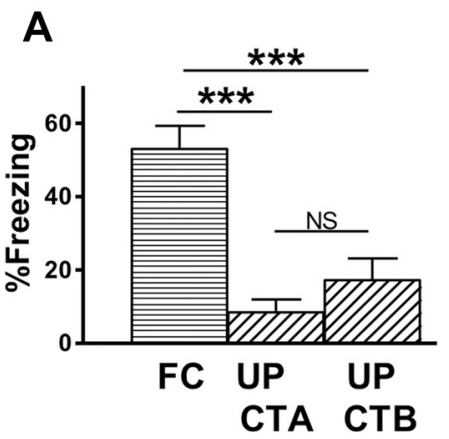

B
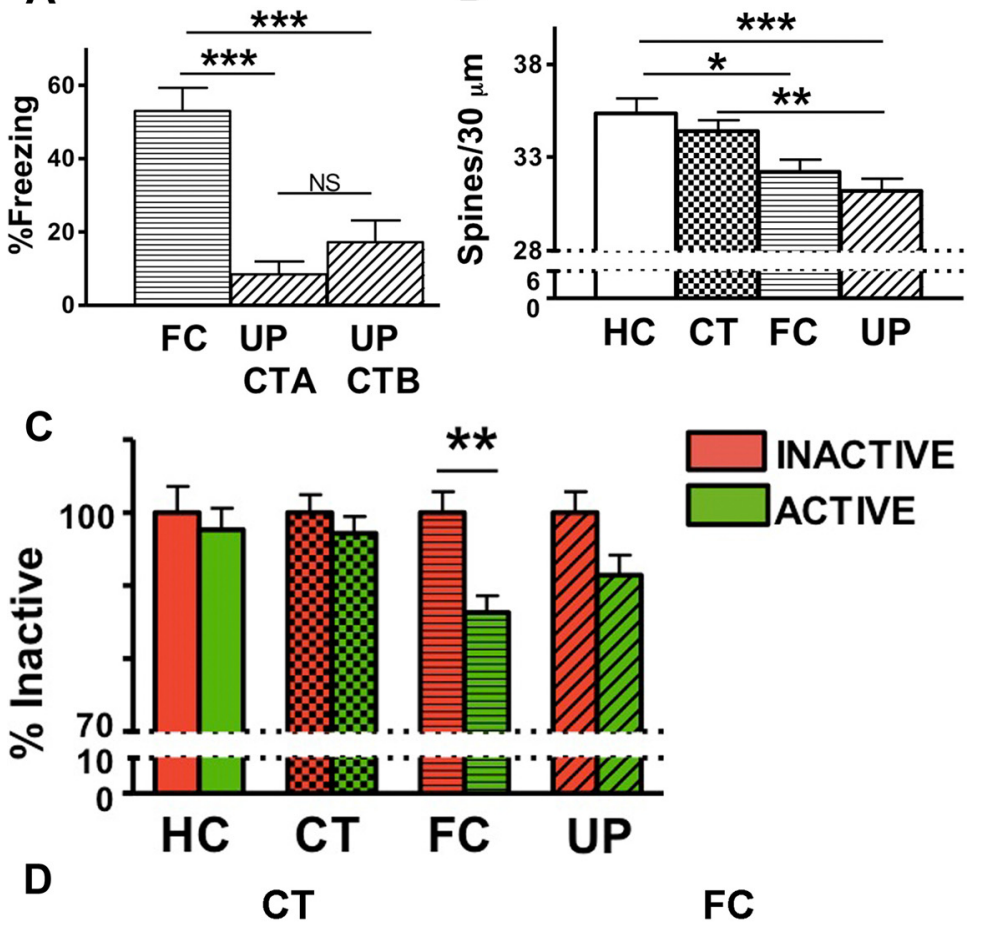

$\square$ INACTIVE $\square$ ACTIVE

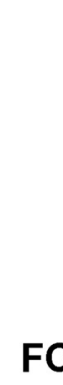

FC
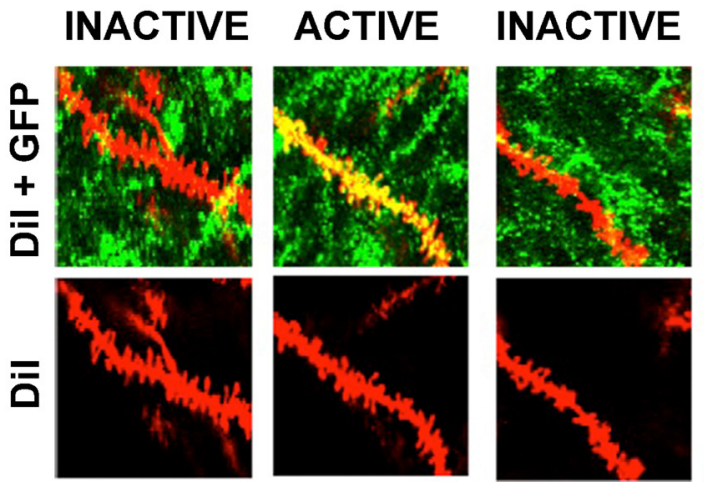

ACTIVE

2. Fear conditioning results in a specific decrease in spine density on active versus inactive neurons. $A$, GFPGluR1 ${ }^{\text {c-fos }}$ mice were fear conditioned in context $B(C T-B)$, and freezing was measured $24 \mathrm{~h}$ later in context $B(F C)$. A separate group of mice were exposed to a distinct context $A$ and then given immediate shock in context $B$ (UP). These animals displayed limited freezing (10-20\%) in either of the two unpaired contexts (UP CT-A, UP CT-B). Data are the mean freezing scores $\pm S E M . B, F C$ resulted in a significant decrease of total spine density, relative to $\mathrm{HC}^{(*} p<0.05$ ). UP resulted in a significant decrease of total spine density relative to homecage $\left.{ }^{* * *} p<0.001\right)$ and relative to $\mathrm{CT}$ exposure alone 0.01). Data are the mean spine number \pm SEM per $30 \mu \mathrm{m}$. C, Comparison of spine density for active and inactive neurons within each learning group. $\mathrm{FC}$ decreased spines on active neurons relative to inactive neurons $\left({ }^{* *} p<0.01\right)$. Active neurons in the $\mathrm{FC}$ group also had significantly fewer spines than either inactive or active neurons of the $\mathrm{HC}$ group 0.05 , respectively) (Table 1). D, Representative images depicting spine density on dendrites of animals exposed to $C T$ and spines); FC ( $n=11$ mice, 3998 spines); UP ( $n=8$ mice, 4241 spines).

ble labeled with GFP-immunoreactive signal and DiI fluorescence were designated as being from active neurons. Dendrites that did not show GFP immunoreactivity were designated as being from inactive neurons. To provide a blinded assessment of spine density by neural activity, GFP immunoreactivity was subtracted from all images before spine counting.

Spines were counted only when they were clearly connected to the shaft of the dendrite. The spine depleted zone arising from the soma was excluded in our analysis. In total, 14,385 spines were counted across 437 $30 \mu \mathrm{m}$ dendritic segments. These spines belonged to 80 inactive and 90 active segments from apical dendrites, and 126 inactive and 141 active segments from basal dendrites. We counted 2015, 4131, 3998, and 4241 spines, respectively, within the $\mathrm{HC}, \mathrm{CT}, \mathrm{FC}$, and UP groups. The HC 


\begin{tabular}{|c|c|c|c|c|c|c|c|c|}
\hline \multicolumn{9}{|c|}{ TOTAL SPINES } \\
\hline \multirow{8}{*}{ Spines $/ 30 \mu \mathrm{m}$} & HC IA & HC A & CT IA & CT A & FC IA & FC A & UP IA & UP A \\
\hline & $35.6+/-1.3$ & $35.1+/-1.0$ & $35.0+/-1.0$ & $34.0+/-0.8$ & $34.9+/-1.0$ & $30.1+/-0.8$ & $32.6+/-0.9$ & $29.8+/-0.9$ \\
\hline & & & & & & $*$ & & $* *$ \\
\hline & & & & & & * & & * \\
\hline & & & & & & ** & & ** \\
\hline & & & & & & * & & * \\
\hline & & & & & & ** & & ** \\
\hline & & & & & & & & \\
\hline
\end{tabular}

Bonferroni's post-hoc analysis of total spine density on active and inactive neurons within experimental protocols. FC caused a decrease in spine density on active compared to inactive neurons of the $\mathrm{FC}$ group. A difference between active and inactive neuron spine density within experimental protocols is not seen for $\mathrm{HC}, \mathrm{CT}$, or UP. Values represent mean spine number $\pm \mathrm{SEM}$ per $30 \mu \mathrm{m}$ dendrite. Statistical comparisons were made relative to group designated by black box within each row. ${ }^{*} p<0.05,{ }^{* *} p<0.01,{ }^{* * *} p<0.001$. IA, Inactive neurons; A, active neurons. HC ( $n=9$ mice, 2015 spines), CT ( $n=10$ mice, 4131 spines), FC ( $n=11$ mice, 3998 spines), UP ( $n=8$ mice, 4241 spines).

group had relatively fewer spines counted due to the low expression of GFP-GluR1 in this group and consequentially fewer dendrites from active neurons.

We categorized as mushroom and thin spines only those spines with a head and neck morphology according to established methods for spine classification (Harris et al., 1992). Those spines without a readily observable head and neck morphology were categorized as stubby spines. Mushroom spines were then further differentiated into spines with a calculated head volume of $\geq 0.1 \mu \mathrm{m}^{3}$, corresponding to a head diameter of approximately $\geq 0.6 \mu \mathrm{m}$. Spines that had two heads connected to a single neck were classified as branched spines. This method is in accordance with established methods for spine type classification (Harris et al., 1992; Bourne and Harris, 2008).

The strength of GFP signal within the stratum radiatum and the stratum oriens of the hippocampal CA1 region was measured with MetaMorph software and quantified as the percentage increase in GFP signal above HC. The increases in GFP staining were analyzed in the hippocampal neurons with a two-tailed $t$ test comparing HC to FC groups.

The effects of our experimental protocols and neural activity on total spine density were analyzed with a factorial ANOVA and Bonferroni's post-hoc test. The effects of our experimental protocols and neural activity on spine morphology were analyzed with a factorial ANOVA and Fisher's post-hoc test After statistical analysis, data examining the relationship of spine changes to neural activity were converted to a percentage of the inactive circuit spine density for each learning group.

\section{Results}

To investigate the relationship of spine changes in learning to patterns of neural activity associated with the learning event, we used the GFP-GluR1 ${ }^{\mathrm{c}-\text { fos }}$ transgenic mouse. The mouse expresses a tetracycline response element-linked GFP-GluR1 fusion protein in an activity-dependent and dox-dependent manner through regulation by a second c-fos-tTA transgene. Discrete windows for mapping brain activity can be opened by keeping mice on dox for several weeks before experiments, and then removing dox from the diet before experimental manipulations (Fig. 1A).

Subjecting GFP-GluR1 ${ }^{c-f o s}$ to CT alone, FC, and UP protocols results in strong GFP-GluR1 expression above HC controls. HC control levels were low, as previously described, but FC resulted in a threefold increase in the percentage of CA1 neurons expressing GFP-GluR1 (Fig. 1B) (Matsuo et al., 2008). This was paralleled by an $\sim 80 \%$ increase in GFP-GluR1 signal within the dendritic layers of CA1 (Fig. $1 B$ ). Costaining hippocampal sections with GFP and DiI allowed us to identify active neurons and compare dendrites from these and from inactive neurons. We could thereby relate the structure of dendrites to the pattern of neural activity during learning (Fig. 1C). Mice kept on dox from weaning through maturity were grouped into HC, CT, FC, or UP experimental protocols at maturity (Fig. 1D), and were removed from dox for $4 \mathrm{~d}$ before training. The $\mathrm{HC}$ experimental group remained within their homecage throughout the experiment. In a separate group of mice, we confirmed that our FC protocol resulted in a strong contextual freezing and that our UP protocol did not (Fig. 2A).

Twenty-four hours after training, animals were killed and brain sections were processed for imaging of dendritic spines. Our experimental protocols significantly affected total spine density (active and inactive neurons combined) with total spine density lower in the experimental groups that received footshocks $\left(F_{(3,429)}=7.1918, p=0.0001\right)(\mathrm{HC}$ vs FC, $p<0.05$; HC vs UP, $p<0.001$; CT vs UP, $p<0.01$ ) (Fig. $2 B$ ).

To determine the relationship of neural activity at the time of training to spine density changes, we dissociated spine changes on dendrites from active $(\mathrm{GFP}+)$ and inactive (GFP-) neurons. Neural activity was associated with lower spine density on active compared with inactive neurons $\left(F_{(1,429)}=10.292, p=\right.$ 0.00144 ). This is presented as the percentage spine density in active neurons relative to inactive neurons within each group (Fig. 2C). The decrease in spine density was found specifically on active relative to inactive neurons of FC animals $\left({ }^{* *} p<0.01\right)$. This change was not seen in HC mice or mice exposed to CT or UP (Fig. 2C,D; Table 1).

Recent findings show that synaptic inputs cluster on active neurons as a mechanism of circuit remodeling associated with learning (Takahashi et al., 2012). We therefore investigated whether spine clustering was seen in our experiment and whether this was selective for active neurons. If this occurred on the active cells of our fear-conditioned group, we hypothesized that clustered inputs would be identified as a discrete population of dendrite segments enriched in spines, occurring amid an overall increase in pruned segments.

We did not see clustering but instead found that the FC resulted in a generalized increase in pruned segments in the active circuit without any evidence of a clustered spine population. As opposed to the FC group, the HC, CT, and UP groups had similar and overlapping spine density distributions for both active and inactive cells (Fig. 3). These data do not suggest that spine changes are anatomically restricted to particular dendritic regions, at least at the $30 \mu \mathrm{m}$ level of resolution.

We further classified spines into mushroom, branched, stubby, and thin morphologies, since distinctive roles for each of 

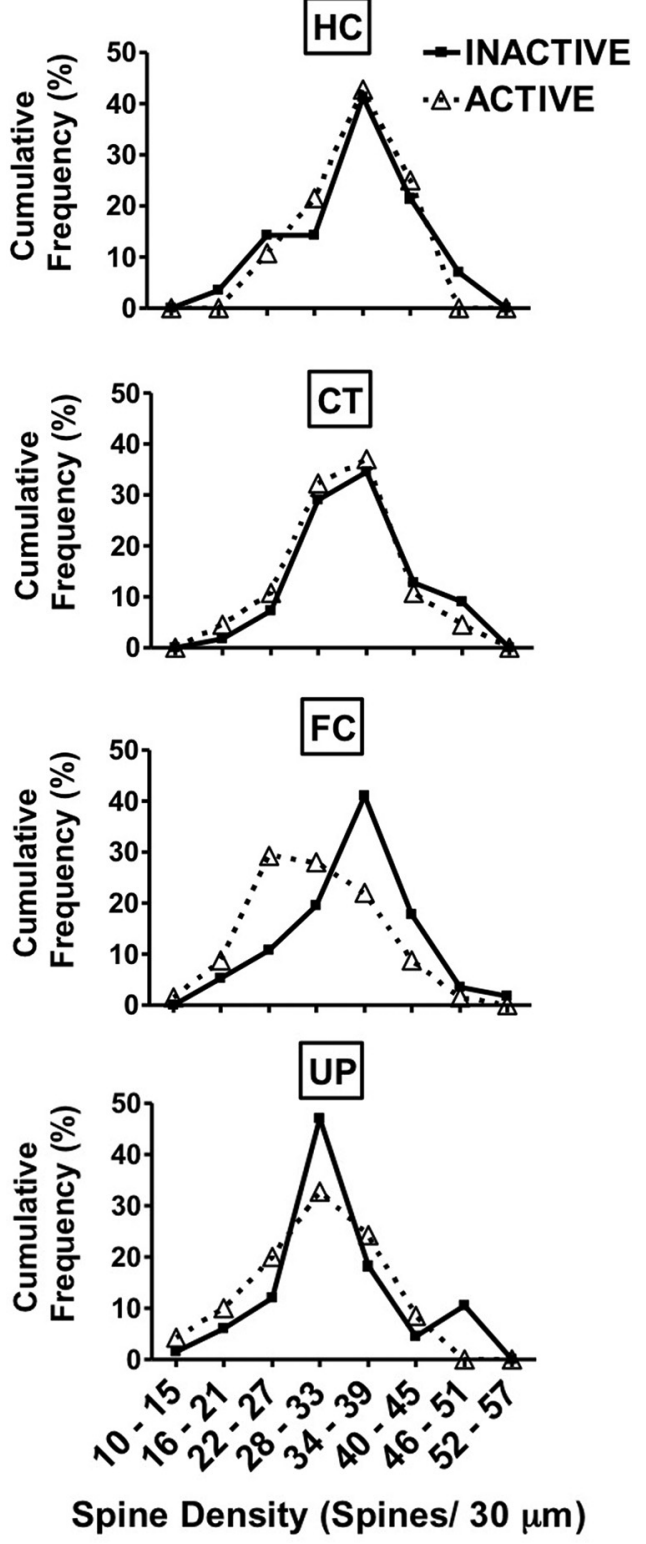

Figure 3. Spine density distributions. FC resulted in a generalized increase in pruned segments in the active circuit without any evidence of a clustered spine population. As opposed to the FC group, the $H C, C T$, and UP groups had similar and overlapping spine density distributions for both active and inactiveneurons. Data represent cumulative percentage frequency of spine densities for each learning group.

these subtypes are implicated in various plasticity paradigms (Fig. $4 A$ ). For example, studies have implicated mushroom spines in memory (Bourne and Harris, 2007; Matsuo et al., 2008), and changes in branched spines have been identified in LTP (Trommald et al., 1996). To assess whether spine elimination is restricted to a specific spine morphology, we analyzed the changes occurring in each morphological type (Fig. 4A).

Our experimental protocols significantly affected mushroom spines (Fig. $4 B$; Table $2 ; F_{(3,429)}=9.9801, p<0.001$ ). CT significantly increased mushroom spines relative to their levels in the HC group $(p<0.05)$. Relative to the CT group, though, there were fewer mushroom spines in the groups that received footshock (FC vs CT, $p<0.05$; UP vs CT, $p<0.001$ ). Between the groups that received a footshock, mushroom spines were lower in the UP compared with the FC group $(p<0.01)$ (Fig. $4 B$ ).
Our protocols also led to changes in mushroom spines when analyzed by their relative density on active and inactive neurons. There were fewer mushroom spines on active compared with inactive neurons of the FC group $(p<0.05)$, but this activityrelated change was not seen in HC mice or mice exposed to UP or CT training (Fig. 4B; Table 2). Thus, while both groups that received a footshock showed reductions in mushroom-type spines compared with CT, in the UP group this was a generalized decrease across neurons, while in the FC group the change was specific to the circuit that was active during learning. This activity-specific decease in mushroom spines accounted for $\sim 27 \%$ of the total spine decrease on active neurons with FC, and was hence representative of activity-specific elimination within the total spine population, but did not account for all of the spine loss. We further examined other spine morphologies for their contribution to activity-specific decreases with learning.

Our protocols significantly affected branched spines $\left(F_{(3,429)}=\right.$ 2.6562, $p=0.04803)$, with more branched spines on the neurons of the UP group compared with mice subject to CT $(p<0.05)$ and FC $(p<0.05)$. Neural activity was associated with lower branched spine density on active compared with inactive neurons $\left(F_{(1,429)}=4.9105 . p=0.02722\right)$. The decrease in branched spine density was specifically found on active but not on inactive neurons of FC and UP animals $(p<0.001$ and $p<0.05$, respectively), and it was not seen in $\mathrm{HC}$ mice or mice exposed to CT (Fig. $4 B$; Table 2).

Experimental protocols led to small but significant changes in stubby and thin spines with fewer stubby spines on neurons of mice in the UP group compared with the HC $(p<0.05)$ and CT groups $(p<0.05)$. Within experimental groups, we did not find activity-specific changes for stubby spine morphology (Fig. 4C; Table 2). FC resulted in a decrease in the thin spines relative to HC $(p<0.05)$. There were fewer thin spines on active neurons compared with inactive neurons of the FC group $(p<0.05)$, but this activity-related change was not seen in $\mathrm{HC}$ mice or mice exposed to UP or CT training (Fig. 4C; Table 2).

\section{Discussion}

Structural changes in dendrites have been proposed as an important mechanism of long-term information storage (Bailey and Kandel, 1993; Trommald et al., 1996; Maletic-Savatic et al., 1999; Yang et al., 2008). The subtle and transient nature of many of these structural changes, however, has called into question whether they accompany long-term memory within the sparsely distributed circuits that are active during learning (Marrone, 2007; De Roo et al., 2008). To study whether long-term memory is associated with spine changes on the neurons active during learning, we used a transgenic mouse that expresses an enduring dendritic marker (GFP-GluR1) under the control of the c-fos promoter.

Our data show that the stress of footshocks, as delivered during FC or UP, resulted in hippocampal CA1 spine loss, consistent with a documented role of stress in decreasing spine density (Michelsen et al., 2007; Chen et al., 2008, 2010, 2012; Bloss et al., 2011; Mucha et al., 2011). However, an important distinction is observed when spine density is analyzed in the active and inactive neural ensembles. With unsignaled footshocks in the UP group, the spine loss was generalized across the active and inactive neurons. In the FC group, the footshock is paired with a specific context to develop an associative fear memory, and spine loss was restricted to the neural population that was active at the time of learning.

These findings corroborate the previous data of others that have shown a role for learning in modifying the effects of stress on hip- 
A
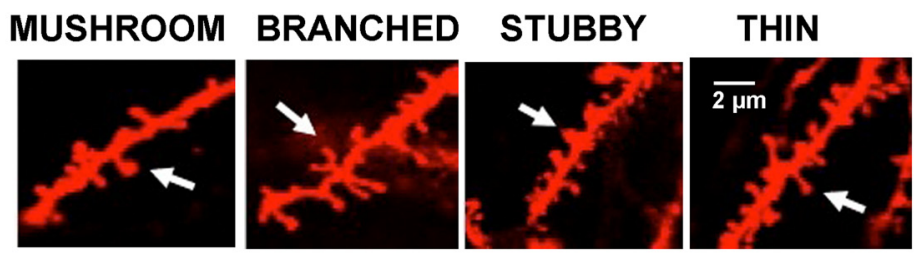

B

BRANCHED
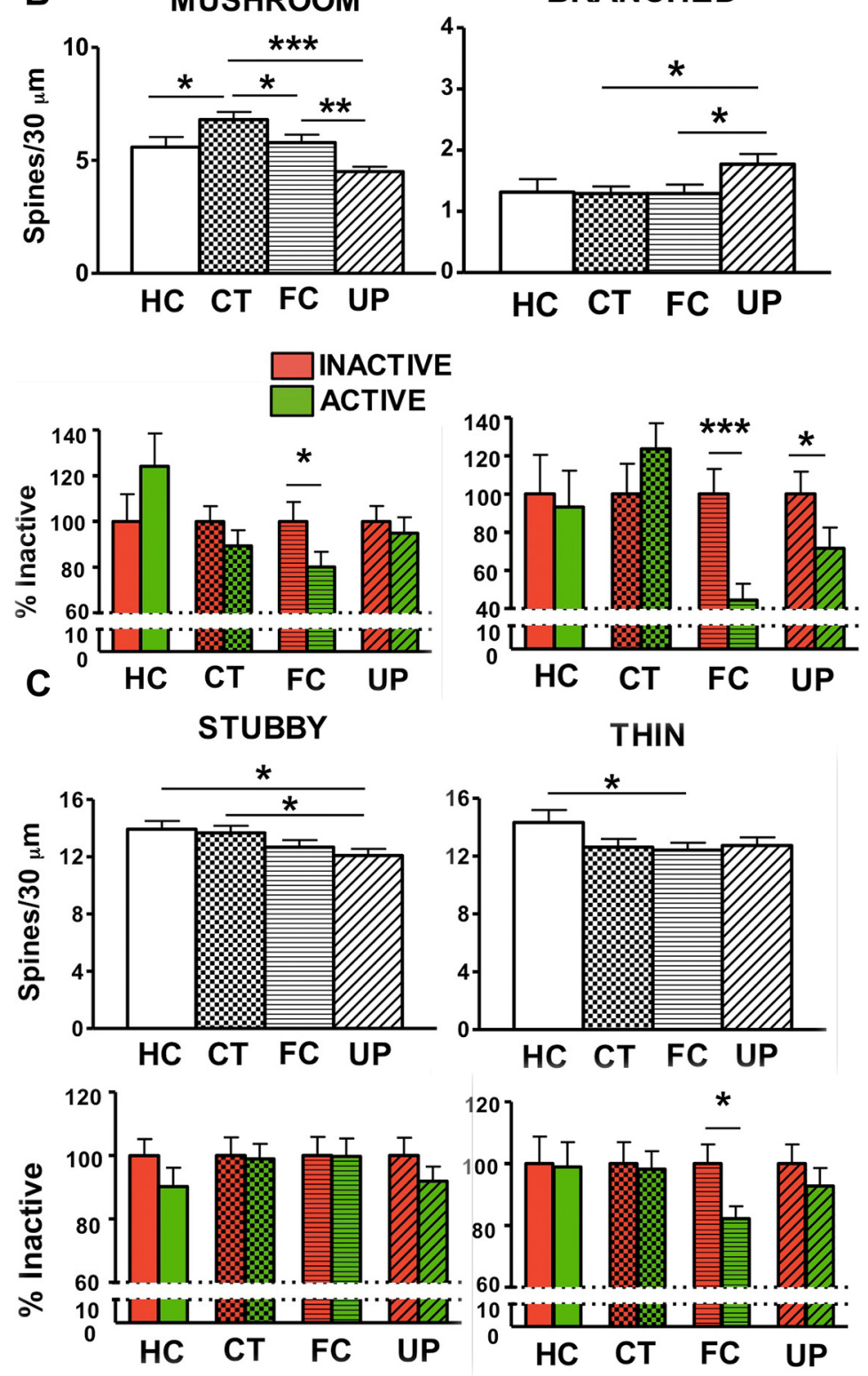

Figure 4. Morphological analysis of spine density changes. $\boldsymbol{A}$, Representative confocal images of thin, stubby, mushroom, and branched spine types. $\boldsymbol{B}, \mathrm{CT}$ resulted in an increase in mushroom spines relative to the $\mathrm{HC}$ group $\left({ }^{*} p<0.05\right)$. $\mathrm{FC}$ and UP resulted in fewer mushroom spines compared with CT (FC vs $C T,{ }^{*} p<0.05$; UP vs $\mathrm{CT},{ }^{* * *} p<0.001$ ). FC resulted in fewer mushroom spines on active neurons compared with inactive neurons $\left({ }^{*} p<0.05\right)$. UP resulted in a small but statistically significant increase in branched spines compared with CT and FC $\left({ }^{*} p<0.05\right)$. FC and UP resulted in fewer branched spines on active neurons compared with inactive neurons ( ${ }^{* * *} p<0.001$ and ${ }^{*} p<0.05$, respectively). C, UP resulted in fewer stubby spines compared with $\mathrm{HC}\left({ }^{*} p<\right.$ $0.05)$ and $\mathrm{CT}\left({ }^{*} p<0.05\right)$. FC resulted in fewer thin spines compared with $\mathrm{HC}\left({ }^{*} p<0.05\right)$. There were fewer thin spines on active neurons of the $\mathrm{FC}$ group $\left({ }^{*} p<0.05\right)$. Data are presented as the mean spine number \pm SEM per $30 \mu \mathrm{m}$ and as the percentage spine density relative to inactive cells within each learning group \pm SEM. pocampal systems (Shors et al., 1989). For instance, LTP is impaired when animals are exposed to the stress of an inescapable footshock. This LTP impairment, though, is much less when this stress is accompanied by escape learning (Shors et al., 1989). The neural processes that subserve these protective effects of learning are poorly understood. Our data suggest that they may be due to a restricted, circuit-specific loss of spines when stress occurs with learning. This is in contrast to a more profound and generalized spine loss that may cause hippocampal impairment when this brain structure experiences an unpredictable and nonassociative stressor.

At the level of spine morphology, one might expect this effect to be most apparent within thin spines since this morphology is considered to be susceptible to pruning with stress-related signaling (Chen et al., 2012). The overall modest response of thin spine morphology to the stressful exposure of footshock, though, is in contrast to what may be hypothesized from these prior studies. These differences may be due to in vitro methods that applied corticotropin-releasing hormone to hippocampal slices and found a decrease in thin spines (Chen et al., 2012), compared with our in vivo experimental paradigm. Alternatively, these differences may be due to different time points of analysis or varying methods for classifying thin spine morphologies.

In contrast to the modest alterations that we found in thin and stubby spines, there were more changes in branched and mushroom spines. Branched spines have been found to change as a result of LTP and environmental enrichment, leading to the proposition that they are an important structural substrate for synaptic plasticity (Geinisman et al., 1989; Trommald et al., 1996; Johansson and Belichenko, 2002). Our data further suggest a unique role for the branched spine morphology in a structural remodeling that accompanies memory, particularly within active hippocampal circuitry.

Although CT training increased the total population of mushroom spines relative to HC levels, perhaps as an adaptation to spatial learning (Moser et al., 1994), FC and UP were conversely found to result in lower mushroom spines compared with CT. Further analysis of mushroom spines by neural activity found that FC eliminated mushroom spines on active neurons.

The role of such activity-specific spine loss with learning is not known. However, it may serve to promote synaptic transmission effi- 
Table 2. Statistical analysis of spine morphologies on active and inactive neurons

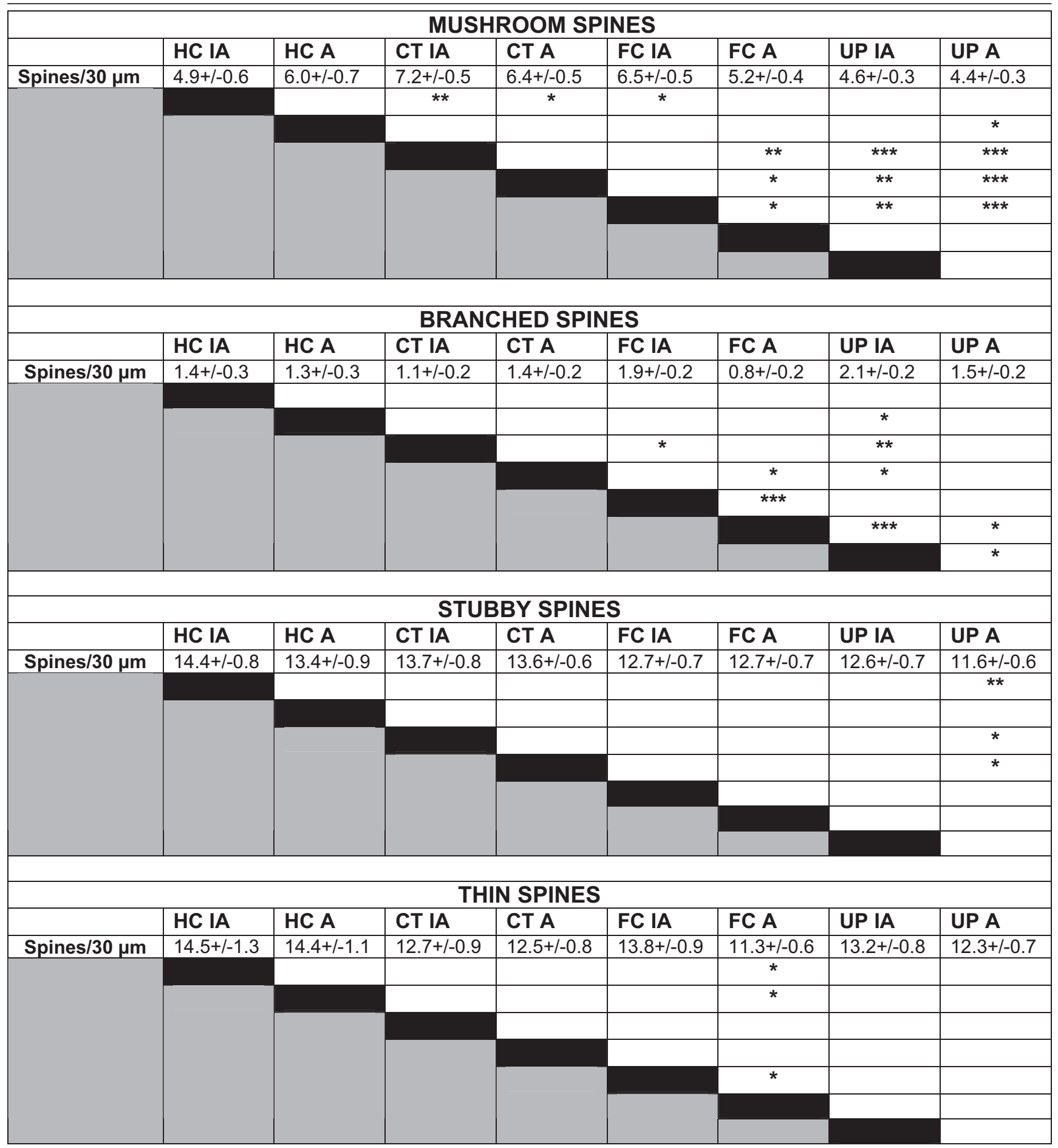

Fisher's post-hoc analysis of spine morphology density on active and inactive neurons within experimental protocols. F( resulted in fewer mushroom spines on active compared to inactiveneurons $\left({ }^{*} p<0.05\right)$, and $\mathrm{FC}$ and UP resulted in fewer branched spines on active compared to inactive neurons ( $*^{* *} p<0.001$ and $p<0.05$, respectively). FC resulted in fewer thin spines on active compared to inactive neurons $(p<0.05)$. Filopodia spines were also measured but were rare and did not reveal any significant changes in response to experimental protocol or neural activity. Values represent mean spine number \pm SEM per $30 \mu \mathrm{m}$ dendrite. Statistical comparisons were made relative to group designated by black box within each row. ${ }^{*} p<0.05,{ }^{* *} p<0.01,{ }^{* * *} p<0.001$. IA, Inactive neurons; A, active neurons.

cacy during future exposure to learned contingencies. Previous studies have shown a circuit-specific insertion of newly synthesized AMPA-type glutamate receptors into spines using a similar fear-conditioning paradigm (Matsuo et al., 2008) (Fig. 5). This AMPA receptor insertion is also induced by artificial stimulation leading to LTP, suggesting that this synaptic strengthening mechanism occurs at concurrently active synapses (Kessels and Malinow, 2009). Conversely, LTD has been associ- ated with spine loss and spike-timing-dependent plasticity protocols show that LTD occurs when presynaptic and postsynaptic activity are discordant (Dan and Poo, 2004; Bastrikova et al., 2008).

Together, this would lead to a strengthening of synapses that are strongly activated by the behavioral contingencies (via AMPA receptor insertion) and a weakening of those synapses that are not driven 


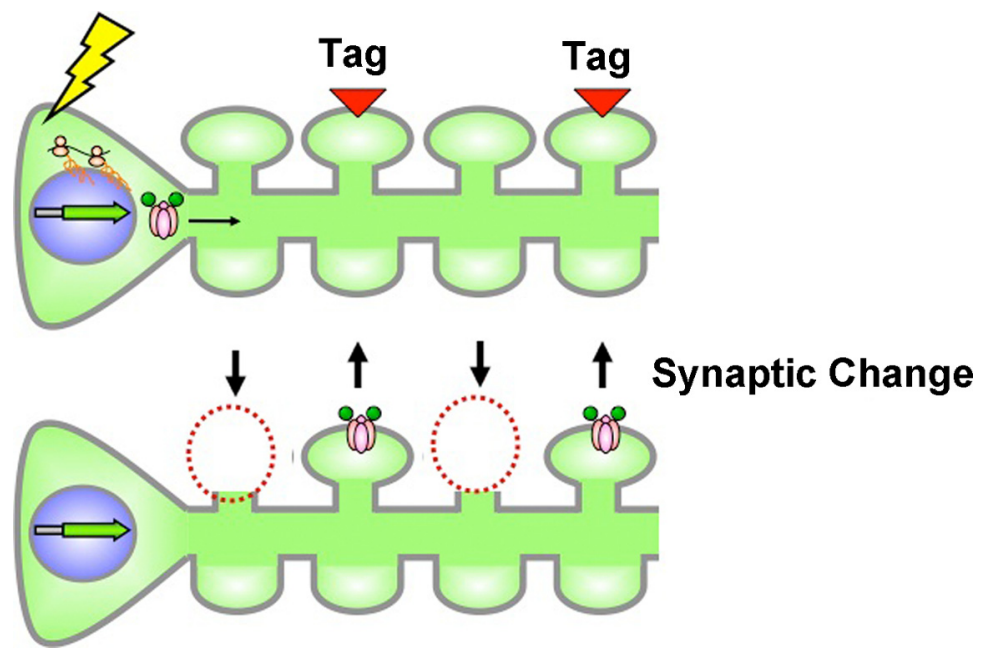

Figure 5. Schematic illustration depicting spine elimination and AMPA insertion as coordinated plasticity mechanisms. Previous studies have shown that fear conditioning produces a molecular tag specifically in mushroom spines, allowing them to integrate newly synthesized AMPA receptors (Matsuo et al., 2008). This synaptic strengthening mechanism could work in conjunction with the spine elimination described here to provide a mechanism for encoding information while retaining a constant level of synaptic input.

\section{References}

Anagnostaras SG, Gale GD, Fanselow MS (2001) Hippocampus and contextual fear conditioning: recent controversies and advances. Hippocampus 11:8-17.

Bailey CH, Kandel ER (1993) Structural changes accompanying memory storage. Annu Rev Physiol 55:397-426.

Bastrikova N, Gardner GA, Reece JM, Jeromin A, Dudek SM (2008) Synapse elimination accompanies functional plasticity in hippocampal neurons. Proc Natl Acad Sci U S A 105:3123-3127.

Bloss EB, Janssen WG, Ohm DT, Yuk FJ, WadsworthS, Saardi KM, McEwen BS, Morrison JH (2011) Evidence for reduced experience-dependent dendritic spine plasticity in the aging prefrontal cortex. J Neurosci 31:7831-7839.

Bourne J, Harris KM (2007) Do thin spines learn to be mushroom spines that remember? Curr Opin Neurobiol 17:381-386.

Bourne JN, Harris KM (2008) Balancing structure and function at hippocampal dendritic spines. Annu Rev Neurosci 31:47-67.

Chen Y, Dubé CM, Rice CJ, Baram TZ (2008) Rapid loss of dendritic spines after stress involves derangement of spine dynamics by corticotropin-releasing hormone. J Neurosci 28:2903-2911.

by the stimuli (reflected in synapse loss) due to discordant presynaptic and postsynaptic activation. These two mechanisms acting together would sculpt the circuit responsiveness to favor activation by the learned contingencies while maintaining a constant level of overall synaptic drive. In favor of this idea, recent findings propose an "inverse" synaptic tagging mechanism whereby weak synapses are silenced within strongly activated circuits to prevent their undesired enhancement (Okuno et al., 2012). Our findings may reflect the structural equivalent of inverse synaptic tagging.

Circuit-specific spine elimination as a result of FC points to an important role for the hippocampus in associative processes underlying this form of learning. These observations indicate that a role for the hippocampus in FC extends beyond an encoder of contextual representations that is ancillary to associative processes localized within the amygdala (Anagnostaras et al., 2001). Our data as well as the data of others (Moita et al., 2004) suggest that the hippocampus plays an important role in integrating context representation with shock.

In summary, the subtle and transient structural changes found in synaptic and behavioral plasticity paradigms have challenged whether or not there are enduring spine modifications that underlie memory (Marrone, 2007; De Roo et al., 2008). Our data point an important locus for such modifications within hippocampal ensembles that are active during learning. These findings are compatible with recent findings showing structural refinement within hippocampal circuitry that is active during learning (Kitanishi et al., 2009a,b). For example, mice that explore a novel enriched environment exhibit fewer spines on activated hippocampal neurons $1 \mathrm{~h}$ later (Kitanishi et al., 2009b). Our data show a similar circuit-specific spine reduction that persists as an enduring structural signature to strong emotional learning. This spine reduction may reflect a stressinduced spine elimination that is focused into circuit-specific structural modifications to refine synaptic connectivity. Collectively, these data imply that dendritic spine perturbations in cognitive disorders impact an intricate system of activity-specific structural reorganization that may be important for long-term memory.
Chen Y, Rex CS, Rice CJ, Dubé CM, Gall CM, Lynch G, Baram TZ (2010) Correlated memory defects and hippocampal dendritic spine loss after acute stress involve corticotropin-releasing hormone signaling. Proc Natl Acad Sci U S A 107:13123-13128.

Chen Y, Kramar EA, Chen LY, Babayan AH, Andres AL, Gall CM, Lynch G, ator CRH involves selective destruction of thin dendritic spines via RhoA signaling. Mol Psychiatry. Advance online publication. Retrieved August 4, 2012. doi: $10.1038 / \mathrm{mp} .2012 .17$.

Dan Y, Poo MM (2004) Spike timing-dependent plasticity of neural circuits. Neuron 44:23-30.

De Roo M, Klauser P, Garcia PM, Poglia L, Muller D (2008) Spine dynamics and synapse remodeling during LTP and memory processes. Prog Brain Res 169:199-207.

Desmond NL, Levy WB (1986) Changes in the numerical density of synaptic contacts with long-term potentiation in the hippocampal dentate gyrus. J Comp Neurol 253:466-475.

Geinisman Y, Morrell F, deToledo-Morrell L (1989) Perforated synapses on double-headed dendritic spines: a possible structural substrate of synaptic plasticity. Brain Res 480:326-329.

Harris KM, Jensen FE, Tsao B (1992) Three-dimensional structure of dendritic spines and synapses in rat hippocampus (CA1) at postnatal day 15 and adult ages: implications for the maturation of synaptic physiology and long-term potentiation. J Neurosci 12:2685-2705.

Holtmaat AJ, Trachtenberg JT, Wilbrecht L, Shepherd GM, Zhang X, Knott GW, Svoboda K (2005) Transient and persistent dendritic spines in the neocortex in vivo. Neuron 45:279-291.

Johansson BB, Belichenko PV (2002) Neuronal plasticity and dendritic spines: effect of environmental enrichment on intact and postischemic brain. J Cereb Blood Flow Metab 22:89-96.

Kelsch W, Lin CW, Mosley CP, Lois C (2009) A critical period for activitydependent synaptic development during olfactory bulb adult neurogenesis. J Neurosci 29:11852-11858.

Kessels HW, Malinow R (2009) Synaptic AMPA receptor plasticity and behavior. Neuron 61:340-350.

Kitanishi T, Ikegaya Y, Matsuki N (2009a) Behaviorally evoked transient reorganization of hippocampal spines. Eur J Neurosci 30:560-566.

Kitanishi T, Ikegaya Y, Matsuki N, Yamada MK (2009b) Experiencedependent, rapid structural changes in hippocampal pyramidal cell spines. Cereb Cortex 19:2572-2578.

Lai CS, Franke TF, Gan WB (2012) Opposite effects of fear conditioning and extinction on dendritic spine remodelling. Nature 483:87-91.

Leuner B, Falduto J, Shors TJ (2003) Associative memory formation inBaram TZ (2012) Impairment of synaptic plasticity by the stress medi- 
creases the observation of dendritic spines in the hippocampus. J Neurosci 23:659-665.

Maletic-Savatic M, Malinow R, Svoboda K (1999) Rapid dendritic morphogenesis in CA1 hippocampal dendrites induced by synaptic activity. Science 283:1923-1927.

Marrone DF (2007) Ultrastructural plasticity associated with hippocampaldependent learning: a meta-analysis. Neurobiol Learn Mem 87:361-371.

Matsuo N, Reijmers L, Mayford M (2008) Spine-type-specific recruitment of newly synthesized AMPA receptors with learning. Science 319:1104-1107.

Michelsen KA, van den Hove DL, Schmitz C, Segers O, Prickaerts J, Steinbusch HW (2007) Prenatal stress and subsequent exposure to chronic mild stress influence dendritic spine density and morphology in the rat medial prefrontal cortex. BMC Neurosci 8:107.

Moita MA, Rosis S, Zhou Y, LeDoux JE, Blair HT (2004) Putting fear in its place: remapping of hippocampal place cells during fear conditioning. J Neurosci 24:7015-7023.

Moser MB, Trommald M, Andersen P (1994) An increase in dendritic spine density on hippocampal CA1 pyramidal cells following spatial learning in adult rats suggests the formation of new synapses. Proc Natl Acad Sci U S A 91:12673-12675.

Mucha M, Skrzypiec AE, Schiavon E, Attwood BK, Kucerova E, Pawlak R (2011) Lipocalin-2 controls neuronal excitability and anxiety by regulating dendritic spine formation and maturation. Proc Natl Acad Sci U S A 108:18436-18441.

Murakami G, Tsurugizawa T, Hatanaka Y, Komatsuzaki Y, Tanabe N, Mukai H, Hojo Y, Kominami S, Yamazaki T, Kimoto T, Kawato S (2006) Comparison between basal and apical dendritic spines in estrogen-induced rapid spinogenesis of CA1 principal neurons in the adult hippocampus. Biochem Biophys Res Commun 351:553-558.
Okuno H, Akashi K, Ishii Y, Yagishita-Kyo N, Suzuki K, Nonaka M, Kawashima T, Fujii H, Takemoto-Kimura S, Abe M, Natsume R, Chowdhury S, Sakimura K, Worley PF, Bito H (2012) Inverse synaptic tagging of inactive synapses via dynamic interaction of Arc/Arg3.1 with CaMKIIbeta. Cell 149:886-898.

Penzes P, Cahill ME, Jones KA, VanLeeuwen JE, Woolfrey KM (2011) Dendritic spine pathology in neuropsychiatric disorders. Nat Neurosci 14:285-293.

Restivo L, Vetere G, Bontempi B, Ammassari-Teule M (2009) The formation of recent and remote memory is associated with time-dependent formation of dendritic spines in the hippocampus and anterior cingulate cortex. J Neurosci 29:8206-8214.

Shors TJ, Seib TB, Levine S, Thompson RF (1989) Inescapable versus escapable shock modulates long-term potentiation in the rat hippocampus. Science 244:224-226.

Takahashi N, Kitamura K, Matsuo N, Mayford M, Kano M, Matsuki N, Ikegaya Y (2012) Locally synchronized synaptic inputs. Science 335:353-356.

Trachtenberg JT, Chen BE, Knott GW, Feng G, Sanes JR, Welker E, Svoboda $\mathrm{K}$ (2002) Long-term in vivo imaging of experience-dependent synaptic plasticity in adult cortex. Nature 420:788-794.

Trommald M, Hulleberg G, Andersen P (1996) Long-term potentiation is associated with new excitatory spine synapses on rat dentate granule cells. Learn Mem 3:218-228.

Wosiski-Kuhn M, Stranahan AM (2012) Transient increases in dendritic spine density contribute to dentate gyrus long-term potentiation. Synapse 66:661-664.

Yang Y, Wang XB, Frerking M, Zhou Q (2008) Spine expansion and stabilization associated with long-term potentiation. J Neurosci 28:5740-5751. 